\title{
Plasticity of the gastrocnemius elastic system in response to decreased work and power demand during growth
}

\author{
Cox, SM ${ }^{1,2}$, DeBoef, $\mathrm{A}^{2,3}$, Salzano, $\mathrm{MQ}^{2,4,5}$, Katugam, $\mathrm{K}^{2}$, Piazza, SJ ${ }^{2}$, Rubenson, $\mathrm{J}^{2,4}$
}

$4{ }^{1}$ The Department of Ecology and Evolutionary Biology, The University of California, Irvine, CA,

$5 \quad{ }^{2}$ Biomechanics Laboratory, Department of Kinesiology, The Pennsylvania State University,

6 University Park, PA, ${ }^{3}$ The Department of Biological Sciences, Georgia Institute of Technology,

7 Atlanta, GA, ${ }^{4}$ Integrative and Biomedical Physiology, The Pennsylvania State University, University

8 Park, PA, United States ${ }^{5}$ Department of Kinesiology, The University of Massachusetts - Amherst,

9 Amherst, MA

10 Abstract

11 Elastic energy storage and release can enhance performance that would otherwise be limited by the

12 force-velocity constraints of muscle. While functional influence of a biological spring depends on

13 tuning between components of an elastic system (the muscle, spring, driven mass, and lever system),

14 we do not know whether elastic systems systematically adapt to functional demand. To test whether

15 altering work and power generation during maturation alters the morphology of an elastic system,

16 we prevented growing guinea fowl (Numida Meleagris) from jumping. At maturity, we compared the

17 jump performance of our treatment group to that of controls and measured the morphology of the

18 gastrocnemius elastic system. We found that restricted birds jumped with lower jump power and

19 work, yet there were no significant between-group differences in the components of the elastic

20 system. Further, subject-specific models revealed no difference in energy storage capacity between

21 groups, though energy storage was most sensitive to variations in muscle properties (most

22 significantly operating length and least dependent on tendon stiffness). We conclude that the 
23 gastrocnemius elastic system in the guinea fowl displays little to no plastic response to decreased

24 demand during growth and hypothesize that neural plasticity may explain performance variation.

\section{Introduction}

26 Taking advantage of storage and release of elastic strain energy can enhance performance that would

27 otherwise be limited by the force-velocity constraints of muscle. The temporal decoupling of energy

28 production from energy delivery permitted by elastic energy storage allows muscles and tendons to

29 produce force effectively over a range of shortening or lengthening speeds. Muscles may generate

30 forces during slow or isometric contractions and elastic recoil augments the rate of energy delivery

31 or absorption during rapid movements [1,2]. By making use of energy storage in the tendon

32 "spring", a muscle tendon unit (MTU) can produce force more economically or with greater power

33 than a muscle alone [2]. Yet, several studies have identified important differences among spring-

34 muscle combinations. Wilson, Lichwark, and colleagues [3,4] showed that the efficiency of an MTU

35 during cyclic loading depends on the tuning of relative muscle and spring properties. For instance,

36 muscle efficiency varies with both fascicle length and tendon stiffness, with the specific optimal

37 efficiency values depending on gait conditions [3]. Several researchers [5-9] have shown that the

38 opposing inertial or drag forces acting on a motor-spring system also influence whether springs

39 enhance performance. Adding further complexity, Sawicki and colleagues [10,11] found that the

40 timing of neural activation of muscle during hopping must be tightly controlled to take advantage of

41 in-series springs. Together, this body of work suggests that understanding the conditions in which

42 spring systems enhance performance may require expanding our focus from the muscle-tendon unit

43 to that of the broader 'elastic system' which includes the muscle (motor), the spring, the resistive

44 forces, and the neural control of the system. The optimal performance of an elastic system may 
require tuning of both morphology and neural control. This approach recognizes the integrated nature of the neuro-musculoskeletal system[12].

The sensitivity of elastic system efficiency to the tuning of its components complicates inferences for how elastic systems systematically adapt to functional demand during maturation. For instance, do growing individuals who regularly perform functions that utilize elastic strain energy develop elastic systems with greater energy storage capacity? This is still unknown because most studies of MTU plasticity have focused on how individual components of elastic systems (neural control [1315], muscle [16-19] and tendon [20-25]) vary with task or training, and how those individual changes influence function of a muscle-tendon unit [10,11,26-28]. Yet, the integrated nature of the elastic system suggests that functional consequences of plasticity are difficult to predict by analyzing elements in isolation $[27,29,30]$. Therefore, the complex nature of the neuromuscular adaptation of elastic systems may require analysis at the system level rather than at the level of individual components.

Here we present a study of the morphological plasticity of an elastic system. Specifically, we ask

59 whether individuals that jump during maturation (an activity requiring elastic energy storage and return [31]) develop elastic systems that more capable of storing elastic strain energy at maturity than those of individuals restricted from jumping. Here we focus on the elastic system most involved in storage and release of elastic energy during jumping[32-36], the gastrocnemius elastic system. We test this by altering the rearing conditions of two groups of guinea fowl (Numida meleagris) across the

64 entire growth period, allowing one group to engage in jump-to-perch behavior and preventing all

65 jumping in the other group. We previously reported that restricted birds in this study showed

66 detriments in jump performance at adulthood [37]. In this manuscript, we aim to link the

67 morphological and functional consequences of our intervention. 
Of the morphology data, we take both an individual-component and systems-level approach to evaluate the plasticity of an elastic system during growth. At the component level, we probe whether our treatment resulted in systematic morphological differences in individual components of

71 the gastrocnemius elastic system between groups. We seek to determine whether components of this

72 elastic system plastically adapt to variations in functional demand during growth. At the systems

73 level, we ask how plastic changes at the component level interact to influence the capacity for elastic

74 energy storage. To do this, we developed subject-specific musculoskeletal models that incorporated

75 experimentally measured morphological properties of each bird's elastic system. With each subject-

76 specific model, we simulated a fully activated muscle contraction under various postures and

77 quantified the resulting tendon energy stored. The purpose of this systems-level analysis was to

78 evaluate the integrated effects of morphological variation.

79 The component-and systems-level analyses serve as a case study for understanding how a particular

80 elastic system changes with functional demand. We also took advantage of the variation within and

81 across groups to ask broader questions about the relationship between form and function in elastic

82 systems. Specifically, we asked which combinations of naturally occurring morphological variation

83 most influence the ability of an elastic system to store energy. Lastly, we probed the extent to which

84 energy storage capacity in the gastrocnemius elastic system correlated with jump performance.

85 We hypothesize that components of an elastic system plastically adapt to variations in functional

86 demand during maturation, resulting in greater energy storage capacity in birds that jump during

87 growth. We predict energy storage capacity will increase linearly with muscle force-generating

88 capacity and inversely with tendon stiffness [38-40]. Finally, we predict that differences in jump

89 performance positively correlate with an animal's ability to store elastic strain energy in the tendon

90 of the gastrocnemius elastic system. 


\section{Methods}

\section{Experimental Protocol}

93 Animals. To study these questions, one-day-old guinea fowl keets (Numida meleagris) were obtained

94 from a regional breeder (Guinea Farm; New Vienna, IA). After a 2-wk brooding period, the keets

95 were pen reared through skeletal maturity ( $>6$ months) in one of two conditions, as we previously

96 described in detail (Cox et al 2020). A control group $(C ; n=8)$ was housed in a large, circular pen

$97\left(3.14 \mathrm{~m}^{2}\right)$ that allowed ample room for locomotion and objects for jumping and perching. The

98 restricted treatment group $(\mathrm{R} ; \mathrm{n}=7)$ were raised a smaller pen $\left(1 \mathrm{~m}^{2}\right.$ at maturity) with low mesh

99 ceilings that prevented jumping. Food and water were available ad libitum (food intake did not differ

100 between groups). Lights were programmed to be on a 12:12-h light-dark cycle. The experimental

101 protocol was approved by Institutional Animal Care and Use Committee at The Pennsylvania State

102 University (IACUC; Ref. \#46435).

103

104

105

106

107
Movement Analysis. As described previously [37], to quantify the influence of pen configuration on the movement patterns of both treatment groups, pens were filmed from above for ten minutes, four times per day, across the growth period (Foscam; C2 1080p HD cameras, Houston, TX). For each bird in the pen, behaviors were tracked and categorized into two states (standing or walking) and three events [short sprint ( $<2 \mathrm{sec})$, hurdle jump ( $<$ body height) and perch jump $(\sim 2 \mathrm{x}$ body height).

\section{Functional Measures}

As described previously [37], jump performance was measured by placing each bird in turn on 6x6 in. force plates (AMTI HE6x6; Watertown, MA, USA) enclosed in a tapered box and encouraging the birds to jump. Jump power was calculated from the instantaneous net vertical ground reaction and the vertical center of mass velocity. Velocity was obtained by integrating the center of mass 
113 acceleration, which was in turn found from the net ground reaction force and the body mass. We

114 calculated jump work by integrating the instantaneous power with respect to time over the course of

115 the jump.

116 Quantification of properties of individual components of the elastic system

117 Specimen muscle architecture preparation. The pelvic limb was separated from the upper body and the left

118 and right legs were then split by sectioning the pelvis at the midline while avoiding muscle

119 attachments. Right limbs were placed into neutral buffered formalin for fixation (10\%) for at least

120 two weeks, while left legs were fresh-frozen and kept at $-20^{\circ} \mathrm{C}$. Right limbs were positioned with

121 joint angles approximating those at mid-swing during running (hip: $30^{\circ}$, knee: $80^{\circ}$, ankle: $125^{\circ}$,

122 within $\left.\pm 2^{\circ}[41]\right)$. Joint angles were confirmed for the fixed limbs using photographs made with a

123 digital camera (Canon EOS550D; Surrey, United Kingdom) and analyzed with ImageJ (National

124 Institutes of Health, Betesda, MD). Left limbs were fresh-frozen and saved for later muscle mass

125 measures.

126 Muscle Analyses. We made measurements of the lateral and medial heads of the gastrocnemius muscle

127 (LG and MG), the muscle group of the MTU thought primarily responsible for storage and release

128 of elastic strain energy during running and jumping [31,36]. The third (intermedia) head of the

129 gastrocnemius only comprises $\sim 10 \%$ of the total mass of the gastrocnemius muscles in this species

130 [42] and thus was not included in the analysis. MG and LG were dissected from the fresh-frozen

131 left limbs and weighed to the nearest 0.1 milligram. The LG and MG were then dissected from the

132 fixed limbs for fascicle length, pennation angle, and sarcomere analysis. LG was first split

133 longitudinally through the mid-belly to view fascicle arrangement. Photographs of whole MG and

134 split LG made with a digital camera (Canon EOS550D) were imported into ImageJ for 
135 measurement of the pennation angle between muscle fascicles and their insertions on the

136 aponeurosis [43].

137 Due to the expected within-muscle heterogeneity of strain [44,45], each muscle was divided into

138 sections for analysis. MG was split into anterior and posterior fascicles [46] and then again split

139 proximally/distally, resulting in four sections. The LG was split into proximal, middle, and distal

140 sections, each spanning one-third the length of the muscle belly. Average pennation angle was found

141 for each section by taking the mean of three angle measurements. Sarcomere lengths for each

142 section were found using the laser diffraction techniques described in [43]. A minimum of three

143 sarcomere length measurements were taken from each muscle fascicle bundle and these

144 measurements were averaged to obtain the mean measured sarcomere length.

145 Optimal fascicle length, Lo, was calculated by multiplying the length of the fascicle by the ratio of

146 optimal sarcomere length of guinea fowl muscle $(2.36 \mu \mathrm{m} ;[46])$ to the mean measured sarcomere

147 length.

148 Pennation angle at optimal fascicle length, $\theta_{O F L}$, was calculated from the average measured

149 pennation angle, $\bar{\theta}$, and the ratio of measure fiber length, $F$, and calculated optimal fascicle length,

$150 \quad \mathrm{~L}_{\mathrm{O}}$ according to the equation [47]:

$$
\theta_{O F L}=\sin ^{-1}\left\{\frac{F_{l} \sin \bar{\theta}}{L_{O}}\right\}
$$

151 Maximum isometric force along the muscle fiber for the MG and LG were calculated from the

152 muscle mass, $m$, optimal fascicle length, $L_{O}$ and muscle density ( $\varrho_{\text {musc }}=1060 \mathrm{~kg} / \mathrm{m}^{3}[48]$ using the

153 specific tension, $f\left(3 \times 10^{5} \mathrm{~N} / \mathrm{m}^{2}\right.$, Rospars and Meyer-Vernet, 2016), according to the equation: 


$$
F_{\text {max }}=\frac{f * m}{\rho_{\text {musc }} L_{0}}
$$

154 We specifically calculated isometric force along the muscle fiber for input into the musculoskeletal model rather than including the influence of pennation angle because pennation angle is a separate

156 input into the OpenSim Millard muscle model (see below for model description), which accounts

157 for the change in pennation angle with muscle

171 Retroreflective markers were placed on dissected

172 limbs to track the relative movement of the tibia

173 and tarsometatarsus in $3 \mathrm{D}$ across a range of joint

174 angles using a 4-camera Motion Analysis system

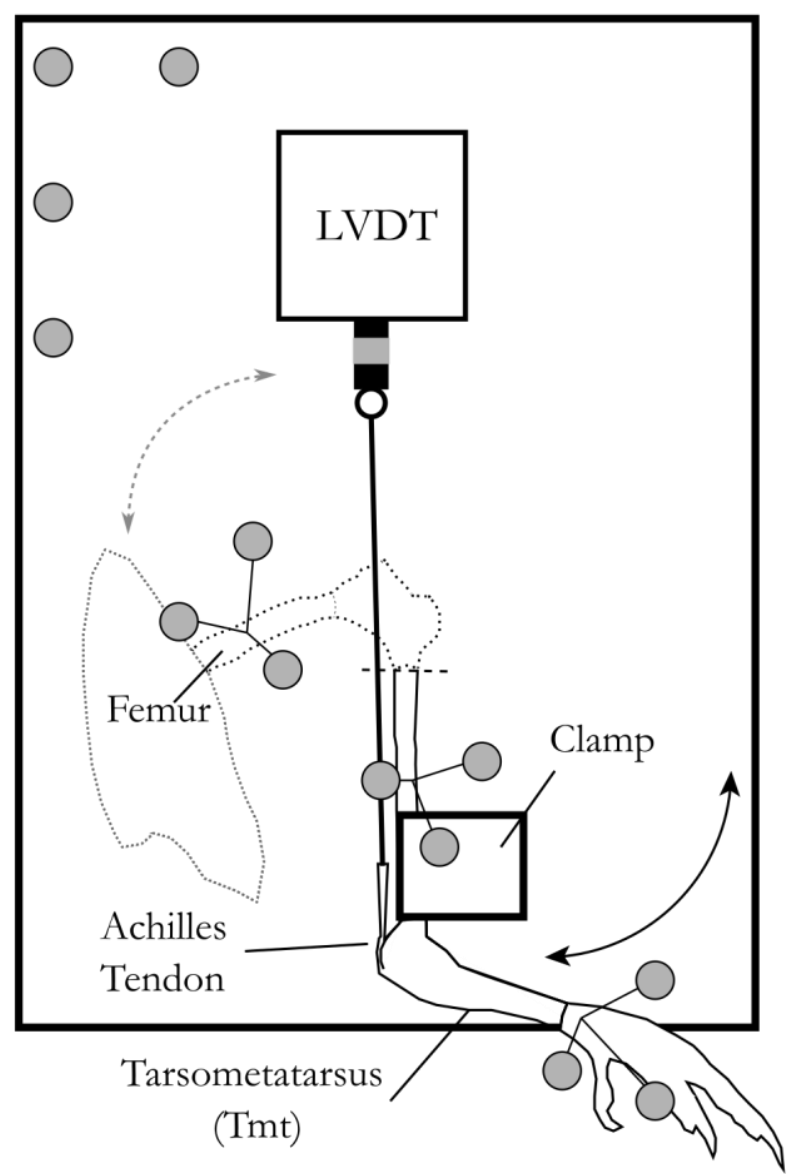

Figure 1: Setup for the tendon travel experiment. The limb was positioned so that the tibiotarsus was held firm by a $3 \mathrm{D}$ printed clamp. In the knee joint motion trial, the femur was rotated to move the knee through its ROM. The tibiotarsus was then cut to remove the proximal portion of the limb, allowing for LVDT to be attached to the Achilles tendon. For tendon travel trials, the TMT was rotated to move the ankle through its ROM. Gray coloring represents retroreflective markers on the limb and LVDT. The dotted line outlines the pelvis femur, and knee, which were removed after the knee joint motion trial. The dashed line represents location at which the tibiotarsus was cut after the knee joint motion trial. Figure adapted from Salzano, 2020. 
175 (300 Hz; Kestrel, Motion Analysis Corporation, Santa Rosa, CA), and automatically synchronized to

176 the linear transducer data within the motion analysis software (Cortex, Motion Analysis

177 Corporation). Joint centres and a mean helical axis were calculated from motion data for each trial

178 and used to calculate flexion angle at the ankle at each timepoint [54]. A cubic spline was fit to the

179 tendon excursion versus flexion angle points using least-squares approximation and tendon

180 excursion was differentiated with respect to angle to estimate moment arm across the measured

181 range of motion $\left(30^{\circ}-90^{\circ}\right)$. Average values are reported in Table 1.

182 Tendon force-length curves. We quantified the tendon force-length properties with material analysis as

183 described in [25]. In short, tendons were detached from the gastrocnemius muscles but left attached

184 at their insertion points on the tarsometatarsus bone. Both the bone and the tendon's proximal end

185 were connected to a material testing machine (858 Mini Bionix II; MTS Systems Corp; Eden Prairie,

186 MN, United States). Samples were mounted vertically using custom clamps on the tendon

187 aponeurosis and the TMT and attached to a 50-pound load cell (MTS Systems Corp; Eden Prairie,

$188 \mathrm{MN}$, United States). The upper clamp gripped the entire aponeurosis of each sample, leaving only

189 the free tendon exposed to loading. The tendon force-length properties were quantified by loading

190 the tendon cyclically (20 cycles) to 4\% strain. The tendon force-length curves were calculated by

191 averaging the data from last 5 cycles of the loading protocol. Tendon force-strain curves were

192 calculated by normalizing displacement by the length of the tendon, $T_{L}$, measured to the nearest 0.1

193 mm with calipers while under zero force in the material testing setup. Average values for tendon

194 stiffness given in Table 1 were calculated from the slope of the tendon force-length curve across the

195 last 50 points measured in the last 5 cycles of trials, at strain between 3 and $5 \%$.

196 Tendon Slack Length. The tendon slack lengths for the LG and MG were estimated from experimental

197 measures as described in Appendix A. Because model based estimates of muscle fiber length in a 
198 given posture are particularly sensitive to the tendon slack length [55-57] and our calculations

199 involved several simplifying assumptions, we further refined our experimental estimates of tendon

200 slack length by fine-adjusting the tendon slack length parameter in the OpenSim model (see

201 Appendix A for experimental tendon slack length measurement and see below and Appendix B for

202 model development). After experimental moment arms and tendon and muscle properties were

203 added to subject specific models, each model was posed in the individual's fixed posture. The

204 model's tendon slack length was adjusted iteratively in the model until the LG and MG normalized

205 fiber lengths were within $1 \%$ of the experimentally measured values. These final values are listed in

206 Table 2.

207 Quantifying the influence of restricted jumping on energy storage capacity

208 We generated a flock of subject-specific musculoskeletal models by modifying the generic model

209 [58] to match experimental values measured for each bird (see Appendix B). With these models, we

210 quantified the capacity of each subject-specific model to store elastic energy in its Achilles tendon

211 across a range of joint postures $\left[\right.$ Ankle $^{\circ}: 31$ to 145, Knee $^{o}:-145$ to -15 , Figure 2 [58])]. At each

212 posture, the simulated LG and MG were activated at $100 \%$ and the muscle-tendon unit was

213 equilibrated with the OpenSim MATLAB equilibrateMuscles $\bigcirc$ function, which adjusts muscle and

214 tendon length such that tendon force and muscle (active and passive) forces balance. The LG and

215 MG insert on the same tendon but, due to OpenSim modeling constraints, these muscles are

216 modeled as having separate tendons. To calculate the stored elastic energy in the combined Achilles

217 tendon, then, we first extracted the resulting force, $f_{a}$, along the tendon for each muscle. These

218 values were summed and the resulting tendon strain in a single tendon, $\in_{T a}$, was found from the

219 inverse of the experimentally measured force-strain curve. 


$$
\varepsilon_{T a}=g^{-1}\left(f_{a}\right)
$$

220 The strain energy stored in the strain of the tendon, PE (eq. 10), was calculated by integrating the

221 tendon force-strain curve from zero to the calculated strain and multiplying that by the tendon

222 sample length, $L_{T}$, as measured at zero strain during the material testing.

$$
P E=L_{T} \int_{0}^{\varepsilon_{T a}} g(\varepsilon) d \varepsilon
$$

223 Simulations of $100 \%$ activation of the LG and MG were performed across the range of

224 experimentally measured joint angles for the ankle and knee joint (Figures 2A\&B). From these

225 simulations, we extracted the maximum elastic energy storage across all postures for each bird and

226 the ankle and knee angles at which the maximum was achieved, and recorded the values in a pre-

227 jump posture (Figure 2C, [31]). Tendon elastic energy stored in the pre-jump posture has been

228 found to be a requirement for the very high power generated in guinea fowl jumping [31,37].

229 Additionally, we recorded the normalized fiber length for each muscle at this posture at zero

230 activation.

\section{Statistical Tests}

232 To determine whether components of the gastrocnemius elastic system change systematically in

233 response to changes in demand, we evaluated the influence of treatment group (restricted vs.

234 control) on each element of morphology measured. This was accomplished using t-tests if the

235 homogeneity of variance assumption test was passed and using a Kruskal-Wallis test by ranks when

236 this criterion was not met. Non-parametric analyses are indicated with an asterisk after the p-value

237 in Tables 1\&2. The threshold for statistical significance was set at 0.005 after a Bonferroni

238 correction for multiple comparisons. Likewise, the relationship between treatment group and elastic 
energy storage capacity was evaluated with a t-test after data passed tests for normality and

240 homogeneity of variance, as described above for evaluation of differences between groups of

241 individual elastic system components.

242 We used stepwise comparison of Akaike information criterion (AIC) values [stepAIC R Mass

243 package [59]] to determine the parameters and coefficients of the full model that best predicted

244 elastic energy storage potential across natural variation of joint postures in preparation for jumps.

245 The full statistical model evaluated included stored strain energy, PE, as a dependent factor and, as

246 potential independent variables, tendon stiffness, tendon $K$, the summed maximum isometric force

247 capacity of LG and MG along the tendon, sumFMax, the average LG and MG optimal fascicle

248 length, $A v O F L$, and starting muscle length at zero activation of the LG and MG in the pre-jump

249 posture, $A v \operatorname{Len} A 0 c$. We included possible interaction terms between muscle force capacity, tendon

250 stiffness and muscle start length (sumMax $\left.F^{*} t e n d o n K^{*} A v \operatorname{Len} A 0 c\right)$ and between optimal fascicle length

251 and tendon stiffness (avOFL*tendonK) following recommendations by Zajac [60] of functional

252 equivalent muscle tendon joint properties. We did not include muscle moment arm or tendon slack

253 length in the statistical model because they both contributed to the starting muscle length at any

254 given joint posture.

255 To quantify the relative explanatory power of morphological variation of any individual element to

256 predict stored energy to a systems level approach, we compared individual parameter models to the

257 best multi-parameter model found by stepwise comparisons described above. The AIC value of the

258 best model was compared to AIC values of models with individual predictors and their relative

259 explanatory power computed [61].

260 The relationship between Achilles tendon elastic energy storage capacity and experimentally

261 measured muscle-mass-normalized peak power output and jump work were both tested with a linear 
262 model with elastic energy storage as the dependent variable and peak power or jump work as the

263 independent variable. See [62] for details on how power and work were calculated from force plate

Table 1: Morphological and functional measures by treatment. All values are given as means $+/$-standard deviations. 'pVal' column lists the p-value of statistical comparisons between groups. Bolded rows show statistically significant differences between groups. $*$ indicates data reproduced from Cox et al. (2020).

\begin{tabular}{llll} 
& Restricted & Control & pVal \\
\hline Total animals & 8 & 8 & \\
Body mass kg & $1.7 \pm 0.11$ & $1.7 \pm 0.14$ & 0.5 \\
Extensor muscle mass kg & $0.239(0.022)$ & $0.257(0.02)$ & 0.18 \\
Average moment arm cm & $0.91 \pm 0.05$ & $0.94 \pm 0.03$ & 0.24 \\
Tendon stiffness kN/m & $48.1 \pm 13$ & $53.5 \pm 10$ & 0.39 \\
Leg length mm & $345 \pm 11$ & $349 \pm 18$ & 0.56 \\
Femur length/leg length & $0.25 \pm 0.01$ & $0.25 \pm 0.01$ & 0.18 \\
Tibia length/leg length & $0.36 \pm 0.01$ & $0.35 \pm 0.01$ & 0.1 \\
Tmt length/leg length & $0.22 \pm 0.01$ & $0.22 \pm 0.01$ & 0.55 \\
Toe length/leg length & $0.17 \pm 0.01$ & $0.17 \pm 0.01$ & 0.63 \\
*Jumps/day during growth & 0 & $194(349)$ & \\
At maturity & & & \\
*Max jump takeoff velocity m/s & $\mathbf{3 . 3} \mathbf{0 . 4 3}$ & $\mathbf{4 . 0 \pm 0 . 3 6}$ & $\mathbf{0 . 0 0 7}$ \\
*Jump work J/kg & $\mathbf{3 7 \pm 9 . 2}$ & $\mathbf{5 0 \pm 8 . 4}$ & $\mathbf{0 . 0 1 3}$ \\
*Peak power W/kg & $\mathbf{7 8 7 \pm 1 6 5}$ & $\mathbf{1 1 7 1 \pm 1 1 7}$ & $\mathbf{3 . 5 e}-\mathbf{4}$ \\
*Peak jump force/body weight N/N & $\mathbf{4 . 7 \pm 0 . 5 4}$ & $\mathbf{6 . 7 \pm 0 . 7 4}$ & $\mathbf{5 e - 5}$
\end{tabular}

264 data.

\section{Results}

266 Variation in individual elements of the elastic system

267 We found no statistically significant differences in any individual morphological property between

268 birds that jumped during growth and those that did not (all $\mathrm{p}>=0.1$, Tables $1 \& 2$ ).

270 Energy storage capacity between groups

271 We found no significant differences in the capacity to store energy in the strain of elastic elements

272 between birds restricted and unrestricted from high power activities during maturation, despite 
274 (Table 3, Figure 3). This held true both at the peak crouched

275 posture before jump initiation (Figure $2 \mathrm{C}, \mathrm{p}=0.43$ ) and at the

276 posture that optimized elastic energy storage (Figure 2D,

$277 \mathrm{pVal}=0.44)$. It should be noted that the optimum posture for

278 elastic energy storage was at the most extended knee angle and the

279 most flexed ankle angle tested and was $\sim 85^{\circ}$ more extended ankle

280 angle than birds used in preparation for a jump. While this more

281 extended posture lengthened the gastrocnemius and increased the

282 energy stored in the Achilles, it shortened the operating length of

283 the knee extensor muscles, reducing their force-generating

284 capacity. In the prejump posture, the shorter gastrocnemius length

285 decreases elastic storage capacity by $12 \%$ for control birds and

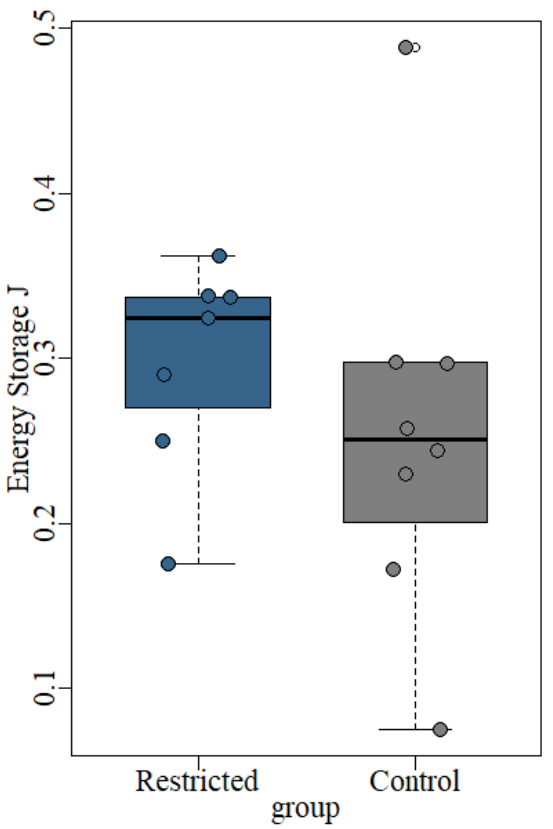

Figure 3: Restricting high power activities does not decrease or significantly influence the capacity to store elastic strain energy. Each dot represents data from one individual.

$28610 \%$ for restricted birds (Table 3) in comparison to the optimal posture for energy storage.

\section{Morphological predictors of elastic energy storage capacity}

Table 2: Muscle morphological data by treatment for the lateral (LG) and medial (MG) gastrocnemius muscles: Muscle mass (Mass g), optimal fascicle length, (Lo mm), Maximum Isometric Muscle force (Max Iso Force N), muscle pennation angle at optimal fiber length (Penn Ang rad), and tendon slack length (Tendon SL $\mathrm{mm}$ ). Morphological measures did not vary between groups. All values are given as means $+/$-standard deviations. pVal column lists the p-value of statistical comparisons between groups.

\begin{tabular}{lllll} 
& & Restricted & Control & pVal \\
\hline LG & Mass g & $9.0 \pm 1.3$ & $9.2 \pm 1.5$ & 0.77 \\
& Lo mm & $24 \pm 2.9$ & $24 \pm 4.1$ & 0.95 \\
& Max Iso Force N & $105 \pm 15$ & $110+-22$ & 0.64 \\
& Pen Ang rad & $0.33 \pm 0.05$ & $0.30 \pm 0.07$ & 0.38 \\
& Tendon SL mm & $135 \pm 7.3$ & $139 \pm 6.5$ & 0.32 \\
MG & Mass g & $11 \pm 1.4$ & $12 \pm 1.8$ & 0.2 \\
& OFL mm & $28 \pm 4.2$ & $29 \pm 3.5$ & 0.81 \\
& Max Iso Force N & $108 \pm 20$ & $118 \pm 29$ & 0.44 \\
& Pen Ang rad & $0.20 \pm 0.03$ & $0.21 \pm 0.03$ & 0.51 \\
& Tendon SL mm & $150 \pm 8.3$ & $150 \pm 9.1$ & 0.97
\end{tabular}


Table 3: Comparison of energy storage capacity and normalized fiber length between restricted and unrestricted birds at maturity across all postures and in the pre-jump posture (shaded grey). Normalized muscle length at the start and end of contraction designated by Average n.Fiber length at A0 and A100 respectively.

\begin{tabular}{clccc} 
& & Restricted & Control & $\mathrm{pVal}$ \\
\cline { 2 - 4 } At max strain & Energy Storage Potential J & $0.3 \pm 0.065$ & $0.26 \pm 0.12$ & 0.44 \\
energy posture & Tendon Strain & $0.12 \pm 0.016$ & $0.098 \pm 0.021$ & 0.031 \\
& Average n.Fiber length at A0 & $1.1 \pm 0.051$ & $0.99 \pm 0.098$ & 0.11 \\
& Average n.Fiber length at A100 & $0.75 \pm 0.051$ & $0.72 \pm 0.054$ & 0.27 \\
& Knee Angle at Max PE & $-131 \pm 1.9$ & $-130 \pm 0$ & 1 \\
& Ankle Angle at Max PE & $-45 \pm 0$ & $-45 \pm 0$ & 1 \\
In pre-jump & Energy Storage Potential J & $0.27 \pm 0.055$ & $0.23 \pm 0.11$ & 0.43 \\
posture (Knee: & Tendon Strain & $0.12 \pm 0.015$ & $0.094 \pm 0.021$ & 0.028 \\
-135 Ankle: & Average n.Fiber length at A0 & $1 \pm 0.05$ & $0.96 \pm 0.097$ & 0.11 \\
$120)$ & Average n.Fiber length at A100 & $0.73 \pm 0.048$ & $0.7 \pm 0.051$ & 0.28
\end{tabular}

290 We found that the amount of energy stored in strain of the tendon was best explained by variation

291 in the average of the passive LG and MG muscle length at activation onset, avLenAO (Table 4).

292 Figure 4A illustrates that tendon strain energy increases at longer starting lengths both between

293 individuals and, even more strikingly within individuals, across postures. Muscle force capacity along

Table 4: Results of models comparing how well variation of individual elastic elements explain variation in elastic storage potential (dark shaded grey regions) and stepwise model comparisons of models with multiple predictors (lighter shaded region) find that muscle operating length at the start of activation (AvLenA0) is most predictive of energy storage, with muscle force capacity, the sum of lateral and medial maximum isometric force (Force), and average gastrocnemius optimal fiber length, avOFL, also significant.. Tendon stiffness, Tendon K, add little to no additional predictive information. A multi-predictor model (Full:, darker shaded region), explained variation in energy storage capacity over 160,000 times better than any individual predictor. Likelihood comparisons between the null and individual models are designated by $\mathrm{L}($ null $\mid \mathrm{x})$, and between the full model and individual predictor models by $\mathrm{L}($ full $\mid \mathrm{x})$. Akaike weights are listed under w(AIC)

\begin{tabular}{|c|c|c|c|c|c|c|c|c|c|c|}
\hline & Coeff & $\begin{array}{l}\text { Adj. } \\
\mathbf{R}^{2}\end{array}$ & pVal & AIC & $\begin{array}{l}\Delta \mathrm{AIC} \\
(1-5)\end{array}$ & $\mathbf{w}(\mathrm{AIC})$ & $\begin{array}{l}L(\text { null } \mid \\
\mathbf{x})\end{array}$ & $\begin{array}{l}\Delta \mathrm{AIC} \\
(2-6)\end{array}$ & w(AIC) & $L($ full $\mid \mathbf{x})$ \\
\hline 1. Null: & & & & -28 & 0 & $1.1 \mathrm{e}-5$ & 1 & & & \\
\hline 2. Force & 0.002 & 0.50 & $2.0 \mathrm{e}-3$ & -37 & -9.4 & $1.2 \mathrm{e}-3$ & 111 & 28 & $1.6 \mathrm{e} 21$ & $1.0 \mathrm{e} 6$ \\
\hline 3.tendon $\mathrm{K}$ & $-1.3 e-8$ & -0.077 & 1 & -26 & 2.0 & $3.1 \mathrm{e}-6$ & 0.37 & 39 & $5.3 \mathrm{e} 18$ & $3.0 \mathrm{e} 8$ \\
\hline 4.AvLenA0 & 0.40 & 0.57 & $6.4 \mathrm{e}-4$ & -40 & -12 & $3.3 e-3$ & 390 & 25 & $5.6 \mathrm{e} 21$ & $2.8 \mathrm{e} 5$ \\
\hline 5. avOFL & -1.5 & 0.26 & $3.1 \mathrm{e}-2$ & -31 & -3.6 & $5.1 e-5$ & 6 & 33 & $8.6 \mathrm{e} 19$ & $1.9 \mathrm{e} 7$ \\
\hline 6.Full: & & 0.93 & $2.3 e-6$ & -65 & & & & 0 & $1.5 \mathrm{e} 27$ & 1 \\
\hline FMax & $6.1 e-3$ & & $3.2 \mathrm{e}-4$ & & & & & & & \\
\hline tendon $\mathrm{K}$ & $1.2 \mathrm{e}-5$ & & 0.02 & & & & & & & \\
\hline AvLenA0 & 0.29 & & $2.0 \mathrm{e}-4$ & & & & & & & \\
\hline avOFL & 0.78 & & 0.07 & & & & & & & \\
\hline $\begin{array}{l}\text { Force* } \\
\text { tendonK }\end{array}$ & $-7.1 e-8$ & & $4.5 \mathrm{e}-3$ & & & & & & & \\
\hline
\end{tabular}


A

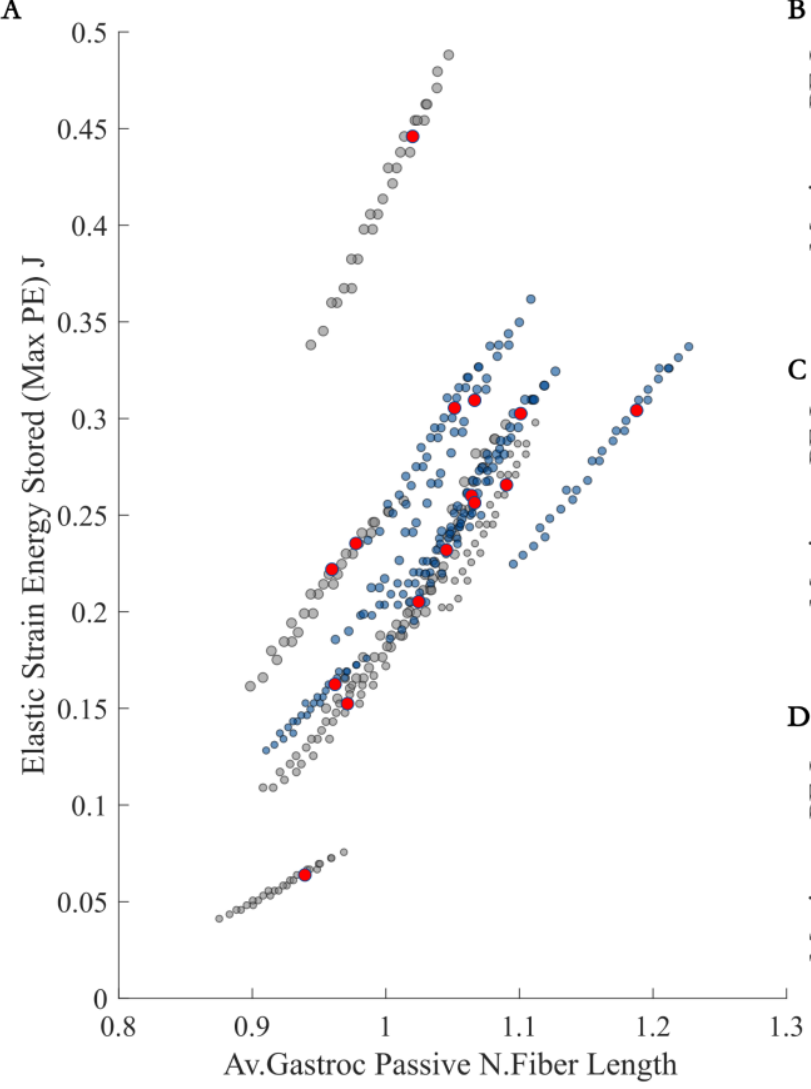

B

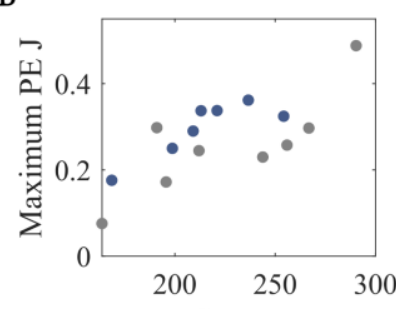

C

Max Iso Gastroc Force N
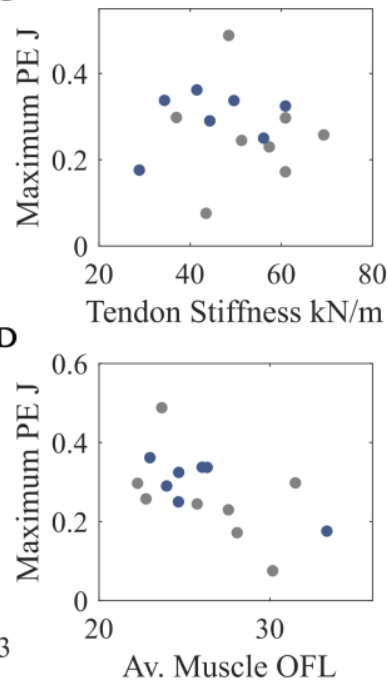

Figure 4: Energy stored in the tendon increases with passive normalized fiber length (length at onset of muscle activation) across different postures (A) and muscle force capacity (B), decreases with muscle optimal fiber length (D), but did not consistently vary with tendon stiffness (C). The variable that most predicts elastic strain energy is muscle normalized fiber length at activation onset (A), with muscles operating at longer lengths enabling greater elastic energy storage. Color designates group (blue: restricted, grey: control). In A), red dots identify the strain energy stored and normalized fiber length in the prejump posture. In B, C, and D each marker represents data from one individual.

294 the tendon was the next most explanatory variable and, like normalized muscle length, shows a

295 positive relationship with energy storage (Figure 4B). In contrast, longer muscle optimal fascicle

296 lengths reduced energy storage (pVal: 0.03, Table 4, Figure 4D) when evaluated as an induvial

297 predictor, but was not a significant factor as a predictor in the full multi-parameter model. Opposite

298 to our predictions, tendon stiffness did not significantly correlate with elastic strain energy when

299 evaluated as an individual predictor ( $\mathrm{pVal}>0.1$ Table 4, Figure 4C), but did improve the explanatory

300 power of a full model. The stepwise AIC comparison of full and reduced models found the sum of

301 LG and MG maximum force capacity along the tendon, sumFMax, average optimal fascicle length, 
302 avOFL, muscle length at activation onset, avLenAO,

303 tendon stiffness and the interaction of tendon

304 stiffness and muscle force capacity as the

305 independent predictors that best correlated with

306 stored elastic strain energy. The relative

307 explanatory power of each predictor following

308 similar patterns as seen in the individual analyses

309 with muscle start length and force capacity

310 showing the greatest predictive power. This full

311 model had an $\mathrm{R}^{2}$ of 0.93 and was over 280,000

312 times more likely to explain the variation in strain

313 energy than any model with only one explanatory

A

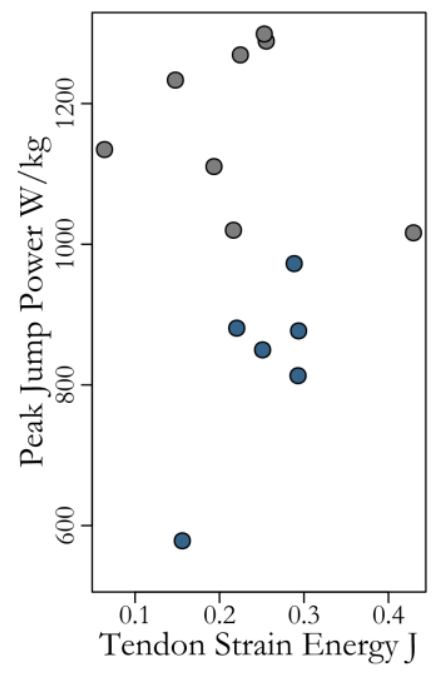

B

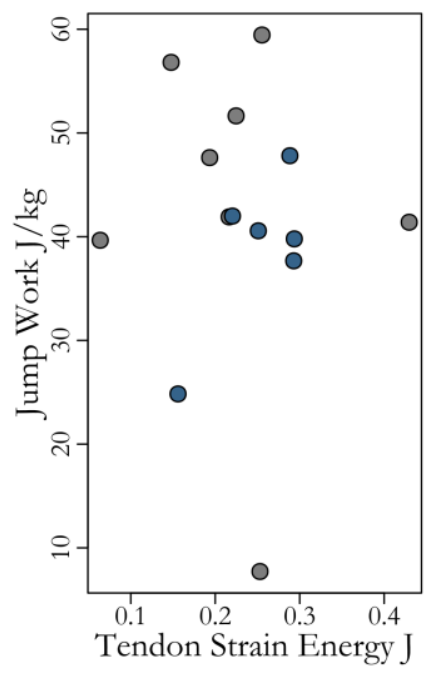

Figure 5: Neither peak jump power nor jump work increase systematically with maximum tendon strain energy across all individuals. Markers designate data from one individual in pre-jump posture and color differentiates treatment groups (grey: control, blue: restricted)

314 variable.

315 Energy storage capacity vs jump performance

316 We found little to no correlation between energy storage capacity predicted by simulations and

317 experimentally collected jump metrics of either muscle mass-specific work or power (Figure 5). A

318 linear model showed no significant relationship between either peak power jump power per $\mathrm{kg}$ of

319 muscle mass capacity $\left(t=-036, p=0.72\right.$, Adj $\left.R^{2}=-0.07\right)$ or jump work $\left(t=-0.05, p=0.96\right.$, Adj $R^{2}=-$

320 0.08) and strain energy in the pre-jump posture. The negative adjusted $\mathrm{R}^{2}$ values for both tests

321 shows that the variation in jump work or peak power explains only a negligible amount of variation

322 in elastic energy storage potential. The scatterplot of standardized predicted values versus

323 standardized residuals for both variables showed that the data met the assumptions of homogeneity

324 of variance and linearity and the residuals were approximately normally distributed. 
327 We found the gastrocnemius elastic system of the guinea fowl robust to variations in locomotor

328 conditions during growth. Neither properties of individual components nor energy storage capacity

329 varied between groups of birds which did and did not jump throughout maturation. Nor did we

330 find any correlation between energy storage capacity and jump performance. Variation in muscle

331 operating length across individuals predicted energy storage capacity better than any fixed

332 morphological property and a systems approach incorporating multiple components was

333 substantially able to predict energy storage capacity better than variation along any individual

334 element.

335 Do components of the gastrocnemius elastic system change systematically in response to 336 changes in power and work demand during growth?

337 Contrary to our predictions, we saw, in general, no systematic changes between the gastrocnemius

338 elastic system in response to decreased demand for high power and work activities during

339 maturation. Surprisingly, birds that were restricted from jumping throughout their entire growth

340 period (Table 1) developed elastic systems that were largely indistinguishable from the control group

341 that jumped, on average, almost 200 times a day and exhibited greater peak jumping power and work

342 at adulthood (Tables 1\&2). Nor did we see differences in body mass, extensor muscle mass, tendon

343 stiffness or leverage (moment arm and/or bone lengths).

345 Two factors may account for why we saw no systematic changes in the elastic system while

346 observations of morphological plasticity in response to changes in functional demand abound [63- 
347 67], even in guinea fowl in particular $[43,68]$. First, several studies suggest that plasticity may vary by

348 life stage, with lower or inconsistent plasticity in growing animals [69-71]). Thus, the inconsistency

349 between the lack of plasticity in our study and the morphological variation found by others suggests

350 that guinea fowl may exhibit lower plasticity during maturation than in adulthood. Fast-growing

351 species, like guinea fowl, might outpace environmental fluctuations with rapid growth and not invest

352 in developmental plasticity [72,73]. A slow growing species (humans for example) might have a

353 selective advantage with greater developmental plasticity. Thus, while our treatment may have been

354 powerful enough to induce morphological changes in adults, rapidly growing guinea fowl may be

355 more robust to environmental perturbations.

357 Second, our results could be consistent with the results of other studies if the plastic response to

358 decreased demand is not inferable from changes in response to increases in demand. For example, it

359 may be that the increase in muscle mass that occurs in response to a certain increase in functional

360 demand is greater than the decrease in muscle mass that occurs in response to the equivalent decrease

361 in demand. Many studies find clear evidence of morphological plasticity, but this was in response to

362 increased mechanical load [24,43,66,68,74,75] and extreme disuse [76-78]. Our intervention,

363 however, eliminated jumping and while maintaining consistent low intensity exercise (i.e. walking)

364 and thus we did not induce chronic offloading, as had been the goal in several previous disuse

365 studies. Our results suggest that there may not be a linear dose-response relationship between

366 changes in functional demand and morphological variation. Instead, as recently suggested [25], there

367 may be a range of variation in demand that is not extreme enough to induce physiological or

368 morphological modification above those under developmental control. If this region of stasis

369 encompasses a wider range of disuse, it could explain both why offloading studies often require

370 extreme disuse, like bedrest or limb immobilization $[76,77,79]$, to induce change and why we found 
371 no systematic morphological changes here. Eliminating jumping may not be an extreme enough

372 disuse signal to induce musculoskeletal plasticity.

374 Thus, while we found no systematic morphological variation when restricting high power activities 375 during maturation, this does not necessarily imply that the morphology of elastic systems does not

376 plastically adapt to variations in functional demand. But it does suggest that there are conditions in

377 which elastic systems may be insensitive to functional variation.

\section{Is the energy storage capacity reduced in individuals that did not jump during growth?}

Despite this lack of consistent morphological variation between our treatment groups, restricted

381 birds generated lower absolute and muscle-mass-specific power and work during jumping. This

382 suggests either that small morphological changes in individual elastic elements compound to alter

383 elastic system function, that variations are significant in other MTU's that we did not quantify, or

384 that behavioral or neural variation account for the difference in jumping performance. Our systems

385 level analysis aimed to specifically address the question of whether morphological variation

386 compound within the elastic system to enable unrestricted birds to store more energy in their

387 Achilles tendon in preparation for a jump. Again, contrary to our predictions, simulations in our

388 subject-specific models resulted in no differences between groups in their ability to store elastic

389 energy. Taken together, the finding of minimal changes to individual muscle-tendon unit

390 components, and no effect on the overall elastic energy storage, could indicate that morphology

391 necessary to enable jumping is highly conserved. This could happen if rapid movements are very

392 critical to fitness, as may be the case for prey animals for whom evasion is critical. 
393 Which type of morphological variation has the greatest influence on energy storage

394 capacity?

395 The first two analyses focus on plasticity of elastic systems and quantified the influence of rearing

396 conditions on the morphology of individual components and how that variation influenced energy

397 storage capacity across treatment groups. Our last two analyses utilize the variation within and

398 across our treatment groups to further probe the relationship between form and function in elastic

399 systems.

400 Evaluating the best predictors of energy storage, we found that muscle properties far outweighed the

401 influence of tendon stiffness. Surprisingly, maximum isometric muscle force, while correlating with

402 energy storage, was not the most important factor. Instead, normalized muscle length at the start of

403 contraction was the best individual predictor of energy storage, with the longest normalized muscle

404 lengths enabling greatest elastic storage [(in agreement with results from $[28,80]]$. This may be

405 because muscles that start contracting on the descending or plateau region of the force length curve

406 increase force capacity as they shorten, resulting in a greater equilibrium force, while muscles starting

407 on the ascending limb of the force-length curve lose force capacity as they shorten against a tendon

408 during an isometric contraction [58]. Further, we were particularly surprised to find that tendon

409 stiffness alone had little to no predictive power of energy storage. Together these data suggest that,

410 between individuals or across an individual's lifetime, the large variation in force capacity due to

411 force-length or force velocity effects may overshadow the influence of variation in tendon properties

412 in determining tendon strain energy. This conclusion is consistent with studies in humans that

413 found no correlation between tendon stiffness and vertical jump height [40]. Yet, this idea runs

414 contrary to the focus on spring properties [23,27,39,81-85] or relative spring and maximal muscle

415 properties [3,75] in many studies that try to connect form and function in elastic systems. Our 
416 results suggest that instead, between or within individuals, elastic energy storage capacity may be

417 more sensitive to variations that alter muscle operating lengths (tendon slack length, optimal fascicle

418 lengths, joint postures) or cross-sectional area than changes in the tendons themselves.

419 Further, our results also highlight the importance of analyzing the components of an elastic system

420 in concert rather than trying to infer performance from variation in one component. Our full model

421 that included both muscle (max muscle force and starting length) and tendon properties explained

422 changes in energy storage capacity over 280,000 times better than variation in any individual

423 property, even when penalizing models for complexity. This, again, emphasizes the limitations of

424 reductionist approaches to understanding how musculoskeletal morphological variation influences

425 the energy storage capacity of an elastic system.

\section{Does elastic energy storage capacity predict peak jump powers and work?}

427 The finding that maximum energy storage capacity of the gastrocnemius elastic system did not

428 predict jump performance further supports the conclusion of the need to switch our focus from

429 individual elements to analyzing the elastic system encompassing both morphology and neural

430 control. Contrary to our expectations, individuals who developed elastic systems capable of storing

431 greater energy in their tendons did not take advantage of that ability to produce more powerful

432 jumps. This suggests that morphology may play a smaller role than neural control in determining

433 contribution of elastic energy storage in jumping. Musculoskeletal morphological variation may not

434 be the main factor limiting jump performance. 
The interaction between tendon and muscle force-length curves may, in part, provide a mechanistic explanation for this disconnect between morphology and performance. Here we include a conceptual diagram to illustrate this (Figure 6). If we plot the muscle and tendon force-length curves on the same figure, it is possible to visualize how they might interact. If the muscle operates on the ascending or plateau region of the force length curve where passive forces are minimal, and we assume that there is no slack in the tendon, a tendon strain of zero will coincide with the muscle

441 length at the start of a contraction. Any tendon strain, then, is equal and opposite to the change in

442 muscle length. During a fixed end contraction, the maximal tendon strain occurs when the tendon

443 force equals the total muscle force of the three heads of the gastrocnemius (empty circles, Figure 6).

444 The tendon strain at equilibrium, then, is dramatically influenced by the length of the muscle when it

445 begins to contract (Figure 6A), reaching higher values for contractions starting at longer muscle

Comparing changes in muscle start length

A

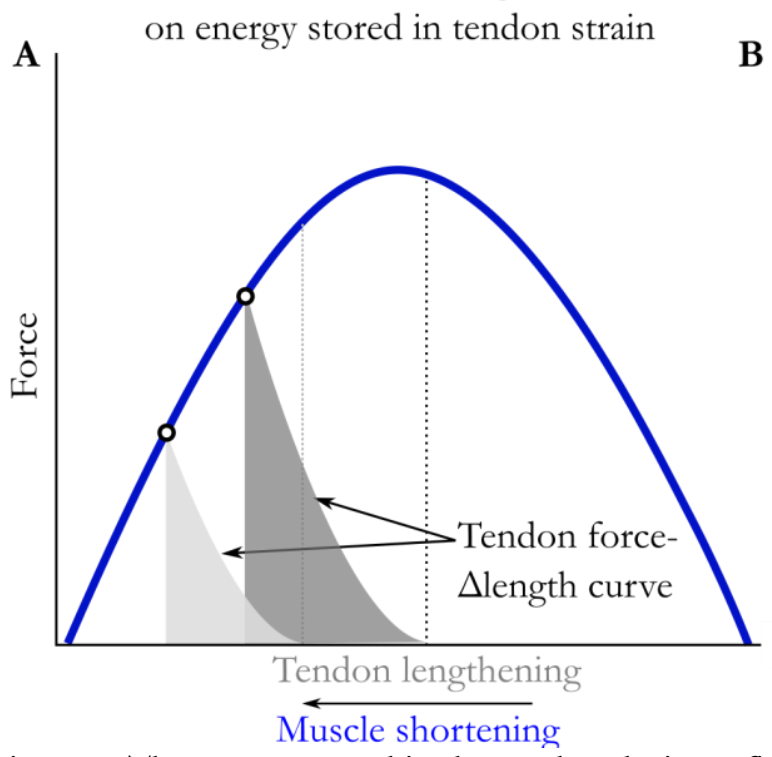

Comparing changes in muscle maximum force capacity on energy stored in tendon strain

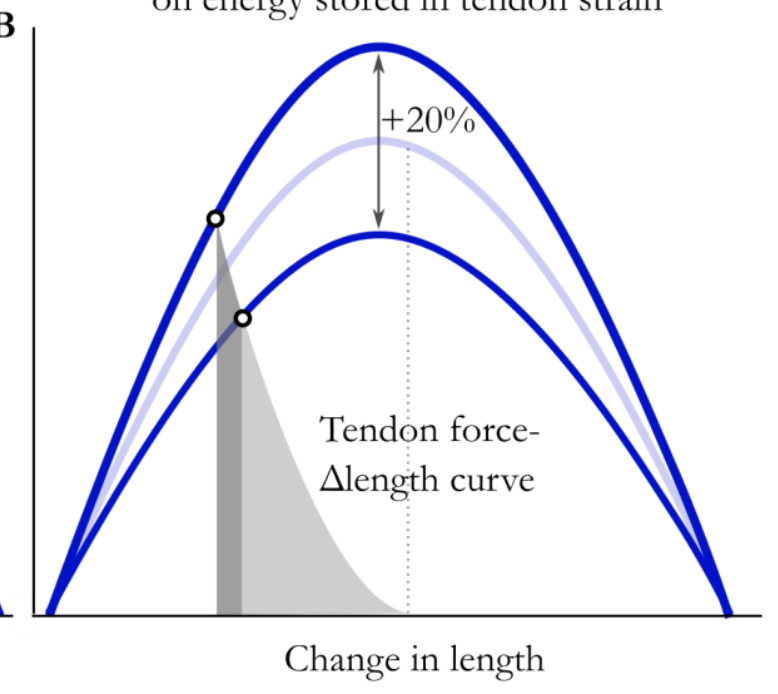

Figure 6: The energy stored in the tendon during a fixed end contraction is constrained by the interaction of the muscle (blue curve) and tendon force- $\Delta$ length curves (grey curve). Since tendon length changes must equal and opposite to muscle length changes, we can plot them at the same scale. Changes in muscle length at the onset of contraction (A) can have a larger influence on energy storage than variations in muscle force capacity (B). The variation in maximum muscle force depicted here represent variation observe in our subjects. Here changes in start length ( $0.9-1.2)$ and muscle force capacity $( \pm 10 \%)$ reflect experimentally measured variation in our population. 
448 of naturally occurring variations in maximal force capacity (Figure 6B). And while muscle operating

449 lengths are constrained by morphology (OFL, pennation angle and moment arms), they are also

450 easily varied with joint angle. Large variations in maximum muscle force could be compensated for

451 by small variations in posture largely under neural control. Thus, as our results suggest, dynamic

452 factors like muscle operating length may influence energy storage more than temporarily fixed

453 musculoskeletal features like maximum muscle force capacity.

454 This suggests that there may be a large range of morphological variation that can be compensated

455 for with neural plasticity and brings up questions of how the two interact. Does variation along a

456 particular morphological axis correlate with systematic changes in neural control? If so, how do

457 individuals search though the neural possibility space? What are the limits of neural compensation?

458 Do we see greater morphological plasticity of the components of elastic systems in conditions that

459 push the limits of neural plasticity?

460 Potential interactions between musculoskeletal and neural variation in elastic energy

461 storage

462 This possible sensitivity of performance to muscle operating lengths also suggests directions for 463 further studies exploring the interaction between morphological and neural or kinematic variation.

464 Here we assumed a uniform pre-jump posture across individuals based on measured average values

465 [31]. Yet our results suggest variations in posture between individuals may play an important role in 466 understanding the relationship between form and function in elastic systems. For instance, one 467 could explore whether individuals with shorter muscle operating lengths adopt a less flexed posture 468 to enable greater tendon energy storage in preparation for a jump. Likewise, the addition of a counter movement proceeding a jump, not common in guinea fowl $[31,37]$ but present in many 
other species [86,87], could minimize influence of muscle constraints on energy storage. This

471 dependence of performance on the interaction between morphology and neural control again

472 reinforces the need to expand our scope from that of the muscle-tendon unit to that of the elastic

473 system.

474 Therefore, while musculoskeletal morphology may set the bounds of possible energy storage,

475 individuals may not operate at their limits of elastic potential. This suggests either a significant

476 behavioral component (restricted birds simply may have not tried as hard to jump) or that there may

477 be benefits to real-time tunability in elastic systems. The jump of a guinea fowl is powered both by

478 tendon recoil and simultaneous muscle work [31], as is common in many larger animals [86-89]. The

479 muscles that load the tendon pre-jump also contract during takeoff to contribute power to the jump.

480 In these hybrid systems, trade-offs between maximizing tendon strain energy and muscle power may

481 explain our findings that the pre-jump posture of guinea fowl did not optimize energy storage in the

482 tendon. Adjusting muscle lengths to maximize tendon strain may hamper muscles fiber work during

483 takeoff. Further, in a complex system such as this, with dozens of individual muscle-tendon units

484 spanning multiple joints and working in concert with direct drive muscles with little tendon, the

485 difference between a great jumper and a good jumper might depend less on the maximal storage

486 capacity of any one muscle tendon unit (i.e. its musculoskeletal morphology) and more on fine

487 adjustments of neural control to harmonize the output of the collective system [90-92].

489 Together, our experimental and modeling analyses suggest that performance advantage of the

490 control birds, who practiced jumping throughout maturation, may lie less in the body's modification

491 of individual elastic elements, and instead, in the fine tuning of neural circuits to coordinate muscle

492 activation timing to take better advantage of what they each possess. While restricting normal

493 locomotor behavior during growth (i.e., eliminating practice) likely leads to deficits in neural control, 
494 neural plasticity is potentially a rapidly reversible pathway to adapt an elastic system to functional

495 variation. Given the potential short timescale of neural plasticity [92,93], greater sensitivity of neural

496 locomotor/movement stimuli could allow the individual to adjust the dynamics of an elastic system

497 during growth without making potentially irreversible changes to morphology that could be

498 detrimental in subsequent stages of growth or in adulthood if environmental conditions or

499 functional demand rapidly change. Thus, one could interpret the results of our study as suggesting

500 that practice during growth may indeed be more related to forming the neural framework for

501 jumping than for forming the musculoskeletal framework. This also suggests the specific hypothesis

502 that individuals restricted from an activity during growth may be capable of reversing the resulting

503 neural deficits with practice later in life.

\section{$504 \quad$ Limitations}

505 Several modeling simplifications could have influenced our results. For instance, the gastrocnemius

506 elastic system is not the only one that could contribute to jump power. While the Achilles is the

507 largest tendon involved, many other digital tendons spanning both the ankle and tarsometatarsus

508 joint have the potential to contribute to jump power but were not included in our analysis

509 Furthermore, changes in the characteristics of muscles spanning proximal joints may also have

510 contributed to the differences in jump power but these muscles were not modeled. Additionally,

511 potentially important dynamic effects were ignored. For simplicity, we simulated the amount of

512 energy stored in the Achilles tendon during fixed-end contractions, where the joint posture was

513 constant as the muscle and tendon dynamically responded to increasing muscle activation.

514 Activating muscles while altering joint posture would alter these dynamics, perhaps amplifying the

515 influence of individual differences in input or output lever lengths or force-velocity effects not

516 apparent from group averages. Likewise, we did not measure and include individual variation in 
517 muscle/aponeurosis passive elastic properties that could significantly alter energy storage [36,94,95].

518 While this is a common approach in musculoskeletal modeling $[50,60,96]$, variation in the

519 aponeurosis and free tendon stiffness [97,98] have the potential to introduce errors [16,99].

520 Another possible limitation was the modeling choice to focus on the potential for an individual to

521 store energy in the strain of their tendon. How that energy is released and how that energy release

522 interacts with synchronous muscle activation could also influence jump performance [5]. Because

523 jumps are likely powered both by tendon recoil and muscle work $[31,80]$, there may be a tradeoff

524 between the work the muscle puts into tendon strain and that which is left available to power the

525 jump during tendon recoil [100]. Future work could involve simulation of jumps in these subject-

526 specific models to assess the contributions of these dynamic factors. Thus, while we found no

527 consistent change in components of the gastrocnemius elastic system due to decreased demand for

528 high power activities during growth, more complex models may provide insight into the ways in

529 which morphological variation constrains performance.

\section{Summary}

531 We found that decreasing the demand for high power and work during growth can influence adult

532 performance but does not necessarily lead to morphological plasticity. We found no difference in

533 energy storage capacity between groups which did and did not jump throughout maturation or any

534 correlation with experimentally measured jump performance. We conclude that gastrocnemius

535 elastic system in the guinea fowl displays little to no morphological plastic response to decreased

536 demand during growth and that neural control of elastic systems may constrain performance more

537 than morphology. 
539 SMC, JR, SJP, MQS contributed to the conception and design of the study. JR, MQS, SJP and SMC

540 developed methodologies and $\mathrm{KK}, \mathrm{AD}, \mathrm{MQS}, \mathrm{SMC}$ collected the data. SMC, MQS analyzed the

541 data and SMC prepared the figures. SMC and JR drafted the initial manuscript. All authors

542 contributed to manuscript editing and approved the final manuscript.

\section{$543 \quad$ Funding}

544 This study was supported in part through a seed grant from the Center for Human Evolution and

545 Diversity, The Pennsylvania State University, and through the National Institute of Arthritis and

546 Musculoskeletal and Skin Diseases of the National Institutes of Health under grant number

547 R21AR071588. The content is solely the responsibility of the authors of this paper and does not

548 necessarily represent the views of the National Institutes of Health.

\section{Conflict of Interest}

550 The authors declare that the research was conducted in the absence of any commercial or financial

551 relationships that could be construed as a potential conflict of interest.

\section{Data Availability}

553 TBD

\section{References}

555 1. Patek SN, Dudek DM, Rosario M V. 2011 From bouncy legs to poisoned arrows: Elastic

556 movements in invertebrates. J. Exp. Biol. 214, 1973-1980. (doi:10.1242/jeb.038596)

557 2. Roberts TJ, Azizi E. 2011 Flexible mechanisms: the diverse roles of biological springs in

$558 \quad$ vertebrate movement. J. Exp. Biol. 214, 353-61. (doi:10.1242/jeb.038588) 
559 3. Lichtwark GA, Wilson AM. 2008 Optimal muscle fascicle length and tendon stiffness for 560 maximising gastrocnemius efficiency during human walking and running. J. Theor. Biol. 252, $561 \quad$ 662-673. (doi:10.1016/j.jtbi.2008.01.018)

562 4. Wilson AM, Van den Bogert AJ, McGuigan MP. 2000 Optimization of the muscle-tendon 563 unit for economical locomotion. In Skeletal muscle mechanics: from mechanism to function (ed W 564 Herzog), pp. 517-47. Hoboken, NJ: John Wiley \& Sons.

565 5. Ilton $\mathrm{M}$ et al. 2018 The principles of cascading power limits in small, fast biological and 566 engineered systems. Science (80-. ). 360. (doi:10.1126/science.aao1082)

6. Richards CT, Sawicki GS. 2012 Elastic recoil can either amplify or attenuate muscle-tendon power, depending on inertial vs. fluid dynamic loading. J. Theor. Biol. 313, 68-78. (doi:10.1016/j.jtbi.2012.07.033)

7. Astley HC, Roberts TJ. 2014 The mechanics of elastic loading and recoil in anuran jumping. J. Exp. Biol. 217, 4372-4378. (doi:10.1242/jeb.110296)

572 8. Astley HC, Roberts TJ. 2012 Evidence for a vertebrate catapult: elastic energy storage in the plantaris tendon during frog jumping. Biol. Lett. 8, 386-9. (doi:10.1098/rsbl.2011.0982)

5749 Galantis A, Woledge RC. 2003 The theoretical limits to the power output of a muscle 575 tendon complex with inertial and gravitational loads. , 1493-1498. (doi:10.1098/rspb.2003.2403)

577 10. Robertson BD, Sawicki GS. 2014 Exploiting elasticity: Modeling the influence of neural 578 control on mechanics and energetics of ankle muscle-tendons during human hopping. J. Theor. Biol. 353, 121-132. (doi:10.1016/j.jtbi.2014.03.010) 
11. Sawicki GS, Robertson BD, Azizi E, Roberts TJ. 2015 Timing matters: tuning the mechanics of a muscle-tendon unit by adjusting stimulation phase during cyclic contractions. J. Exp. Biol.

12. Nishikawa K et al. 2007 Neuromechanics : an integrative approach for understanding motor control. 47, 16-54. (doi:10.1093/icb/icm024)

13. Gillis GB, Biewener AA. 2001 Hindlimb muscle function in relation to speed and gait: in vivo patterns of strain and activation in a hip and knee extensor of the rat (Rattus norvegicus). J. Exp. Biol. 204, 2717-2731.

14. Lutz GJ, Rome LC. 1996 Muscle function during jumping in frogs. I. Sarcomere length change, EMG pattern, and jumping performance. Am. J. Physiol. 271, C563-70.

15. Kao P-C, Lewis CL, Ferris DP. 2010 Short-term locomotor adaptation to a robotic ankle exoskeleton does not alter soleus Hoffmann reflex amplitude. J. Neuroeng. Rehabil. 7, 33. (doi:10.1186/1743-0003-7-33)

16. Lieber RL, Roberts TJ, Blemker SS, Lee SSM, Herzog W. 2017 Skeletal muscle mechanics, energetics and plasticity. J. Neuroeng. Rehabil. 14, 108. (doi:10.1186/s12984-017-0318-y) Polypterus senegalus reared in a terrestrial environment. J. Exp. Biol. 220, 3406-3410. (doi:10.1242/jeb.162909) 
601 19. Iadecola C, Anrather J, Medical WC. 2015 Dramatic changes in muscle contractile and

602 structural properties after two Botulinum toxin injections. Muscle and Nerve 52, 649-657.

603 (doi:10.1038/nm.2399.The)

604

20. Bohm S, Mersmann F, Tettke M, Kraft M, Arampatzis A. 2014 Human Achilles tendon plasticity in response to cyclic strain : effect of rate and duration., 4010-4017.

606 (doi:10.1242/jeb.112268)

21. Docking SI, Cook J. 2019 How do tendons adapt? Going beyond tissue responses to understand positive adaptation and pathology development: A narrative review. J. Musculoskelet. Neuronal Interact. 19, 300-310.

22. Eliasson P, Fahlgren A, Pasternak B, Aspenberg P. 2007 Unloaded rat Achilles tendons continue to grow, but lose viscoelasticity. J. Appl. Physiol. 103, 459-463. (doi:10.1152/japplphysiol.01333.2006)

613 23. Bohm S, Mersmann F, Arampatzis A. 2019 Functional adaptation of connective tissue by 614 training. Dtsch. Z. Sportmed. 70, 105-109. (doi:10.5960/dzsm.2019.366)

615 24. Kubo K, Morimoto M, Komuro T, Yata H, Tsunoda N, Kanehisa H, Fukunaga T. 2007 Effects of Plyometric and Weight Training on Muscle-Tendon Complex and Jump Performance. Med. Sci. Sport. Exerc. 39, 1801-1810. (doi:10.1249/mss.0b013e31813e630a)

618 25. Katugam K, Cox SM, Salzano MQ, De Boef A, Hast MW, Neuberger T, Ryan TM, Piazza SJ,

8. (doi:10.3389/fbioe.2020.00994)

622 26. Mayfield DL, Launikonis BS, Cresswell AG, Lichtwark GA. 2016 Additional in-series 

during fixed-end contractions. J. Exp. Biol. 219, jeb.143123. (doi:10.1242/jeb.143123)

27. Albracht K, Arampatzis A. 2006 Influence of the mechanical properties of the muscle-tendon unit on force generation in runners with different running economy. Biol. Cybern. 95, 87-96. (doi:10.1007/s00422-006-0070-z)

28. Rosario M V, Sutton GP, Patek SN, Sawicki GS. 2016 Muscle - spring dynamics in timelimited, elastic movements. Proc. R. Soc. B 283, 20161561. (doi:10.6084/m9.figshare.c.3462654.)

29. Zajac FE. 1992 How musculotendon architecture and joint geometry affect the capacity of muscles to move and exert force on objects: A review with application to arm and forearm tendon transfer design. J. Hand Surg. Am. 17, 799-804. (doi:10.1016/0363-5023(92)90445-U)

634 30. Ettema GJC. 1996 Elastic and length-force characteristics of the gastrocnemius of the hopping mouse (Notomys alexis) and the rat (Rattus norvegicus). J. Exp. Biol. 199, 12771285. during jumping requires storage and release of elastic energy. J. Exp. Biol. 208, 3293-3302. (doi:10.1242/jeb.01764)

640 32. Alexander R. 1968 Animal Mechanics. University of Washington Press.

641 33. Biewener AA, Mcgowan C, Card GM, Baudinette R V. 2004 Dynamics of leg muscle 642 function in tammar wallabies ( M . eugenii) during level versus incline hopping., 211-223. (doi:10.1242/jeb.00764) 
644 34. Walmsley B, Hodgson JA, Burke RE. 1978 Forces produced by medial gastrocnemius and 645 soleus muscles during locomotion in freely moving cats. J. Neurophysiol. 41, 1203-1216. (doi:10.1152/jn.1978.41.5.1203)

35. Farris DJ, Lichtwark GA, Brown NAT, Cresswell AG. 2016 The role of human ankle plantar flexor muscle-tendon interaction and architecture in maximal vertical jumping examined in vivo. J. Exp. Biol. 219, 528-534. (doi:10.1242/jeb.126854)

36. Arellano CJ, Konow N, Gidmark NJ, Roberts TJ. 2019 Evidence of a tunable biological spring: Elastic energy storage in aponeuroses varies with transverse strain in vivo. Proc. R. Soc.

37. Cox SM, Salzano MQ, Piazza SJ, Rubenson J. 2020 Eliminating high-intensity activity during growth reduces mechanical power capacity but not submaximal metabolic cost in a bipedal animal model. J. Appl. Physiol. 128, 50-58. (doi:10.1152/japplphysiol.00679.2019)

38. Biewener A a, Baudinette R. 1995 In vivo muscle force and elastic energy storage during steady-speed hopping of tammar wallabies (Macropus eugenii). J. Exp. Biol. 198, 1829-41.

39. Biewener AA, Roberts TJ. 2000 Muscle and tendon contributions to force, work, and elastic energy savings: a comparative perspective. Excersice Sport Sci. Rev. 28, 99-107. (doi:10916700) properties of tendon structures on jump performance in humans. , 2090-2096.

662 41. Rubenson J, Marsh RL. 2009 Mechanical efficiency of limb swing during walking and running in guinea fowl ( Numida meleagris ). J. Appl. Physiol. 106, 1618-1630. 
665 42. Rubenson J, Henry HT, Dimoulas PM, Marsh RL. 2006 The cost of running uphill : linking

666

667

668

669

670

671

672

673

674

675

676

677

678

679

680

681

682

683

684

685

686

organismal and muscle energy use in guinea fowl (Numida meleagris ). J. Exp. Biol. 209, 2395-2408. (doi:10.1242/jeb.02310)

43. Salzano MQ, Cox SM, Piazza SJ, Rubenson J. 2018 American Society of Biomechanics Journal of Biomechanics Award 2017: High-acceleration training during growth increases optimal muscle fascicle lengths in an avian bipedal model. J. Biomech. 80, 1-7. (doi:10.1016/j.jbiomech.2018.09.001)

44. Azizi E, Deslauriers AR. 2014 Regional heterogeneity in muscle fiber strain: the role of fiber architecture. Front. Physiol. 5, 303. (doi:10.3389/fphys.2014.00303)

45. Ahn AN, Monti RJ, Biewener AA. 2003 In vivo and in vitro heterogeneity of segment length changes in the semimembranosus muscle of the toad. J. Physiol. 549, 877-888.

(doi:10.1113/jphysiol.2002.038018)

46. Carr JA, Ellerby DJ, Marsh RL. 2011 Differential segmental strain during active lengthening in a large biarticular thigh muscle during running. J. Exp. Biol. 214, 3386-95. (doi:10.1242/jeb.050252)

47. Buchanan TS, Lloyd DG, Manal K, Besier TF. 2004 Neuromusculoskeletal modeling: Estimation of muscle forces and joint moments and movements from measurements of neural command. J. Appl. Biomech. 20, 367-395. (doi:10.1016/j.bbi.2008.05.010)

48. Mendez J, Keys A. 1960 Density and Composition of Mammalian Muscle. Metabolism, 9, 184-188. - References - Scientific Research Publishing. See https://www.scirp.org/(S(lz5mqp453edsnp55rrgjct55))/reference/ReferencesPapers.aspx?Re ferenceID=1219605 (accessed on 19 August 2020). 
49. Rospars JP, Meyer-Vernet N. 2016 Force per cross-sectional area from molecules to muscles: A general property of biological motors. R. Soc. Open Sci. 3. (doi:10.1098/rsos.160313)

50. Millard M, Uchida T, Seth A, Delp SL. 2013 Flexing Computational Muscle: Modeling and Simulation of Musculotendon Dynamics. J. Biomech. Eng. 135, 021005. (doi:10.1115/1.4023390)

51. Spoor CW, van Leeuwen JL. 1992 Knee muscle moment arms from MRI and from tendon travel. J. Biomech. 25, 201-206. (doi:10.1016/0021-9290(92)90276-7)

52. Landsmeer JM. 1961 Studies in the anatomy of articulation. 1. The equilibrium of the ‘intercalated’ bone. Acta Morphol. Neerl. Scand. 3, 287-303.

53. Salzano M. 2020 Musculoskeletal Plasticity in Response to Loading History during Growth. The Pennsylvania State University.

54. Lewis GS, Sommer HJ, Piazza SJ. 2006 In vitro assessment of a motion-based optimization method for locating the talocrural and subtalar joint axes. J. Biomech. Eng. 128, 596-603. (doi:10.1115/1.2205866)

55. De Groote F, Van Campen A, Jonkers I, De Schutter J. 2010 Sensitivity of dynamic simulations of gait and dynamometer experiments to hill muscle model parameters of knee flexors and extensors. J. Biomech. 43, 1876-1883. (doi:10.1016/j.jbiomech.2010.03.022)

56. Ackland DC, Lin YC, Pandy MG. 2012 Sensitivity of model predictions of muscle function to changes in moment arms and muscle-tendon properties: A Monte-Carlo analysis. J. Biomech. 45, 1463-1471. (doi:10.1016/j.jbiomech.2012.02.023) 
model parameters. J. Biomech. 39, 2055-2063. (doi:10.1016/j.jbiomech.2005.06.005)

709 58. Cox SM et al. 2019 The Interaction of Compliance and Activation on the Force-Length Operating Range and Force Generating Capacity of Skeletal Muscle: A Computational Study using a Guinea Fowl Musculoskeletal Model. Integr. Org. Biol. 1, 1-20. (doi:10.1093/iob/obz022)

59. Venables WN, Ripley BD. 2002 Modern Applied Statistics with S. 4th edn. New York: SpringerVerlag.

60. Zajac FE. 1989 Muscle and tendon: properties, models, scaling, and application to biomechanics and motor control. Crit. Rev. Biomed. Eng. 17, 359-411.

61. Wagenmakers E-J, Farrell S. 2004 AIC model selection using Akaike weights. Psychon. Bull. Rev. 11, 192-6.

62. Cox S, Salzano M, DeBoef A, Piazza S, Rubenson J. 2019 Decreased physical activity during growth reduces peak force capacity but not running economy in a bipedal animal model. In International Society of Biomechanics, Calgary, Canada.

(doi:10.1249/01.MSS.0000099092.83611.20) 
729 65. Fitts RH, Widrick JJ. 1996 Muscle Mechanics: Adapations with exercise-training. Exerc. Sport $730 \quad$ Sci. Rev. 24, 427-474.

731 66. Mcdonagh MJN, Davies CTM. 1984 Adaptive response of mammalian skeletal muscle to 732 exercise with high loads. Eur. J. Appl. Physiol. 52, 139-155.

733 67. Wisdom KM, Delp SL, Kuhl E. 2015 Use it or lose it: multiscale skeletal muscle adaptation to 734 mechanical stimuli. Biomech. Model. Mechanobiol. 14, 195-215. (doi:10.1007/s10237-014-0607-3)

735 68. Buchanan CI, Marsh RL. 2001 Effects of long-term exercise on the biomechanical properties 736 of the Achilles tendon of guinea fowl. J Appl Physiol 90, 164-171.

737 69. Legerlotz K, Marzilger R, Bohm S, Arampatzis A. 2016 Physiological Adaptations following 738 Resistance Training in Youth Athletes-A Narrative Review. Pediatr. Exerc. Sci. 28, 501-520. (doi:10.1123/pes.2016-0023)

740 70. Johnston IA. 2006 Environment and plasticity of myogenesis in teleost fish. J. Exp. Biol. 209, 2249-2264. (doi:10.1242/jeb.02153)

742 71. Aucouturier J, Baker JS, Duché P. 2008 Fat and carbohydrate metabolism during submaximal 743 exercise in children. Sport. Med. 38, 213-238. (doi:10.2165/00007256-200838030-00003)

744 72. Dewitt TJ, Sih a, Wilson DS. 1998 Costs and limits of phenotypic plasticity. Trends Ecol. Evol. $745 \quad 13,77-81$.

746 73. Snell-Rood EC. 2012 Selective processes in development: Implications for the costs and 747 benefits of phenotypic plasticity. Integr. Comp. Biol. 52, 31-42. (doi:10.1093/icb/ics067)

748 74. Atherton PJ, Smith K. 2012 Muscle protein synthesis in response to nutrition and exercise. J. 749 Physiol. 590, 1049-1057. (doi:10.1113/jphysiol.2011.225003) 
750 75. Mersmann F, Charcharis G, Bohm S, Arampatzis A. 2017 Muscle and tendon adaptation in

751

752

753

754

755

756

757

758

759

760

761

762

763

764

765

766

767

768

769

770

771

adolescence: Elite volleyball athletes compared to untrained boys and girls. Front. Physiol. 8.

(doi:10.3389/fphys.2017.00417)

76. Bajotto G, Shimomura Y. 2007 Determinants of Disuse-Induced Skeletal Muscle Atrophy: Exercise and Nutrition Countermeasures to Prevent Protein Loss. J. Nutr. Sci. Vitaminol. (Tokyo). 52, 233-247. (doi:10.3177/jnsv.52.233)

77. Clark BC. 2009 In vivo alterations in skeletal muscle form and function after disuse atrophy. Med. Sci. Sports Exerc. 41, 1869-1875. (doi:10.1249/MSS.0b013e3181a645a6)

78. Campbell EL, Seynnes OR, Bottinelli R, McPhee JS, Atherton PJ, Jones DA, Butler-Browne G, Narici M V. 2013 Skeletal muscle adaptations to physical inactivity and subsequent retraining in young men. Biogerontology 14, 247-259. (doi:10.1007/s10522-013-9427-6)

79. Bebout DE, Hogan MC, Hempleman SC, Wagner PD. 1993 Effects of training and immobilization on VO2 and DO2 in dog gastrocnemius muscle in situ. J. Appl. Physiol. 74, $1697-703$.

80. Azizi E, Roberts TJTTJ. 2010 Muscle performance during frog jumping: influence of elasticity on muscle operating lengths. Proc. A R. Soc. B 277, 1523-1530.

(doi:10.1098/rspb.2009.2051)

81. Waugh CM, Blazevich AJ, Fath F, Korff T. 2012 Age-related changes in mechanical properties of the Achilles tendon. J. Anat. 220, 144-155. (doi:10.1111/j.14697580.2011.01461.x)

82. Khayyeri H, Blomgran P, Hammerman M, Turunen MJ, Löwgren A, Guizar-Sicairos M, Aspenberg P, Isaksson H. 2017 Achilles tendon compositional and structural properties are 
83. Fletcher JR, MacIntosh BR. 2018 Changes in Achilles tendon stiffness and energy cost following a prolonged run in trained distance runners. PLOS One 13, 1-17. (doi:10.1371/journal.pone.0202026)

84. Proske U, Morgan DL. 1987 Tendon stiffness: Methods of measurement and significance for the control of movement. A review. J. Biomech. 20, 75-82. (doi:10.1016/0021-9290(87)902697)

85. Waugh CM, Korff T, Fath F, Blazevich AJ. 2014 Effects of resistance training on tendon mechanical properties and rapid force production in prepubertal children. J. Appl. Physiol. 117, 257-266. (doi:10.1152/japplphysiol.00325.2014)

86. Kubo K, Kawakami Y, Fukunaga T. 1999 Influence of elastic properties of tendon structures on jump performance in humans. J. Appl. Physiol. 87, 2090-2096. (doi:10.1152/jappl.1999.87.6.2090) insects. Philos. Trans. R. Soc. Lond. B. Biol. Sci. 347, 235-248. (doi:10.1098/rstb.1995.0024) bullfrogs. J. Exp. Biol. 206, 2567-2580. (doi:10.1242/jeb.00452) 
793 91. Enoka RM. 1997 Neural Adapations with Chronic physical activity. J. Biomech. 30, 465-475.

794 92. Adkins DL, Boychuk J, Remple MS, Kleim JA. 2006 Motor training induces experience795 specific patterns of plasticity across motor cortex and spinal cord. J. Appl. Physiol. 101, 17761782. (doi:10.1152/japplphysiol.00515.2006)

93. Yoxon E, Welsh TN. 2019 Rapid motor cortical plasticity can be induced by motor imagery training. Neuropsychologia 134, 107206. (doi:10.1016/j.neuropsychologia.2019.107206)

94. Huijing PA, Ettema GJ. 1988 Length-force characteristics of aponeurosis in passive muscle and during isometric and slow dynamic contractions of rat gastrocnemius muscle. Acta Morphol. Neerl. Scand. 26, 51-62. tendon and aponeuroses compliance on the force-length relationship. Med. Biol. Eng. Comput.

96. Seth A et al. 2018 OpenSim : Simulating musculoskeletal dynamics and neuromuscular control to study human and animal movement. Comput. Biol. 14, e1006223.

807 97. Finni T. 2006 Structural and functional features of human muscle-tendon unit. Scand. J. Med. Sci. Sport. 16, 147-158. (doi:10.1111/j.1600-0838.2005.00494.x)

809 98. Ettema GJC, Huijing PA. 1989 Properties of the tendinous structures and series elastic component of EDL muscle-tendon complex of the rat. J. Biomech. 22, 1209-1215.

812 99. Epstein M, Wong M, Herzog W. 2006 Should tendon and aponeurosis be considered in series? J. Biomech. 39, 2020-2025. (doi:10.1016/j.jbiomech.2005.06.011) 
814 100. Sutton GP, Mendoza E, Azizi E, Longo SJ, Olberding JP, Ilton M, Patek SN. 2019

815 Integrative and Comparative Biology Why do Large Animals Never Actuate Their Jumps

816 with Latch- Mediated Springs ? Because They can Jump Higher Without Them. , 1-10.

$817 \quad($ doi:10.1093/icb/icz145)

818 101. Winter D. 2009 Biomechanics and motor control of human movement. 4th edn. Hoboken, NH: John

$819 \quad$ Wiley \& Sons, Inc.

820 102. Gollapudi SK, Lin DC. 2009 Experimental determination of sarcomere force-length

821 relationship in type-I human skeletal muscle fibers. J. Biomech. 42, 2011-2016.

$822 \quad$ (doi:10.1016/j.jbiomech.2009.06.013)

823

824 Appendix A

825 Estimation of tendon slack length from experimental measures

826 Tendon slack length was estimated from experimental measures of muscle and tendon morphology

827 as follows. First, the maximum isometric force along the tendon was calculated from the maximum

828 force along the fiber and the pennation angle at optimal fascicle length, $\theta_{O F L}$, according to the

829 equation:

$$
F_{\max T}=F_{\max } \cos \theta_{O F L}
$$

830 The passive force of the muscle exerted on the tendon in the experimentally measured posture was

831 found from the normalized passive muscle force as a function of normalized fiber length curve, 
$832 f_{n p}(n F l)[101,102]$. This was first scaled, for each muscle, by the maximum isometric force along

833 the tendon, $F_{\max }$,

$$
f_{\text {musc } P}=F_{\operatorname{maxT}} f_{n p}(n F l)
$$

834 By normalizing the experimentally measured average fiber length by the muscle's optimal fascicle

835 length, we could calculate the normalized fiber length of the muscle in the fixed posture, $n \mathrm{Fl}$,

836 allowing us to solve equation (4) for the passive force, $f_{m u s c P}$, each muscle exerted on the tendon in

837 the experimental posture. Since the three heads of the gastrocnemius attach to the Achilles tendon,

838 the passive force of each muscle was calculated separately and summed. As the gastrocnemius

839 intermedia head makes up $\sim 10 \%$ of the total gastrocnemius muscle by volume, the passive

840 contribution of this muscle was not experimentally determined for each bird but was estimated from

841 values previously collected [58]. The passive force exerted by the muscle must be balanced by an

842 equal tendon force, thus, the summed passive muscle forces equal the passive force the tendon

843 experienced in the experimental posture.

844 The MTU lengths, $L_{M T U}$, were measured on the fixed limbs by digitizing the three-dimensional

845 paths of the MG and LG from their origins on the tibiotarsus and femur, respectively, to the

846 insertion of the Achilles tendon on the hypotarsus. This approach inherently includes the

847 aponeurosis in the overall tendon length. Digitizing was done using a digitizing arm (Microscribe

848 3DX, Immersion, San Jose, CA). The MTU path was described by 11 points. The linear distances

849 along the MTU path were summed to obtain an overall MTU length. This experimentally measured

850 MTU length, $L_{M T U}$, is the sum of the measured fiber length, $L_{M}$, the tendon's slack length, $L_{T}$, and

851 length change in the tendon (tendon stretch) due to passive muscle fiber force. The length change 
852 in the tendon due to passive muscle fiber force can be described as the tendon strain, $\varepsilon_{T}$, times its

853 tendon slack length, $L_{T 0}$.

$$
L_{M T U}=L_{M}+L_{T}+L_{T} \varepsilon_{T}
$$

854 The strain in the tendon due to the passive muscle fiber force, $\varepsilon_{T}$, was calculated using the

855 experimentally measured tendon force-displacement curve. The tendon force-displacement curve

856 was normalized by tendon length to generate a force-strain curve.

$$
f_{T}=g\left(\varepsilon_{T}\right)
$$

857 The strain at which the tendon force is equal to the passive fiber force can then be found from the 858 inverse of equation (6) and the passive muscle force, $f_{M P}$.

$$
\varepsilon_{T}=g^{-1}\left(f_{M P}\right)
$$

859 The tendon slack length, for each muscle, then, can be calculated from equations (5) and (7).

$$
L_{T 0}=\frac{L_{M T U}-L_{M}}{\left(1+\varepsilon_{T}\right)}
$$

\section{Appendix B}

\section{Development of subject specific models}

863 To perform system level analyses, we modified the generic OpenSim guinea fowl model [58] to

864 generate subject-specific models for each individual. First, the generic model was scaled to match

865 the measured bone lengths and body mass for each bird and saved as distinct models. In each 
866 subject specific model, the generic LG and MG maximum isometric force, pennation angle, optimal

867 fascicle length and tendon slack length were modified to match the experimentally measured and

868 calculated properties.

869 The moment arms of the LG and MG acting at the ankle was fit to experimental values by adjusting

870 the of the size and orientation of the cylindrical wrapping surface for the Achilles at the ankle.

871 During the trial-and-error fitting process, the radius, translation, and rotation of the wrap surface

872 was modified, and the resulting moment arm was compared to the experimentally collected data at

873 31-34 points across the experimental range with a mean moment arm normalized root mean square

874 of the error (Figure S1A) of 0.009 \pm 0.007.

A

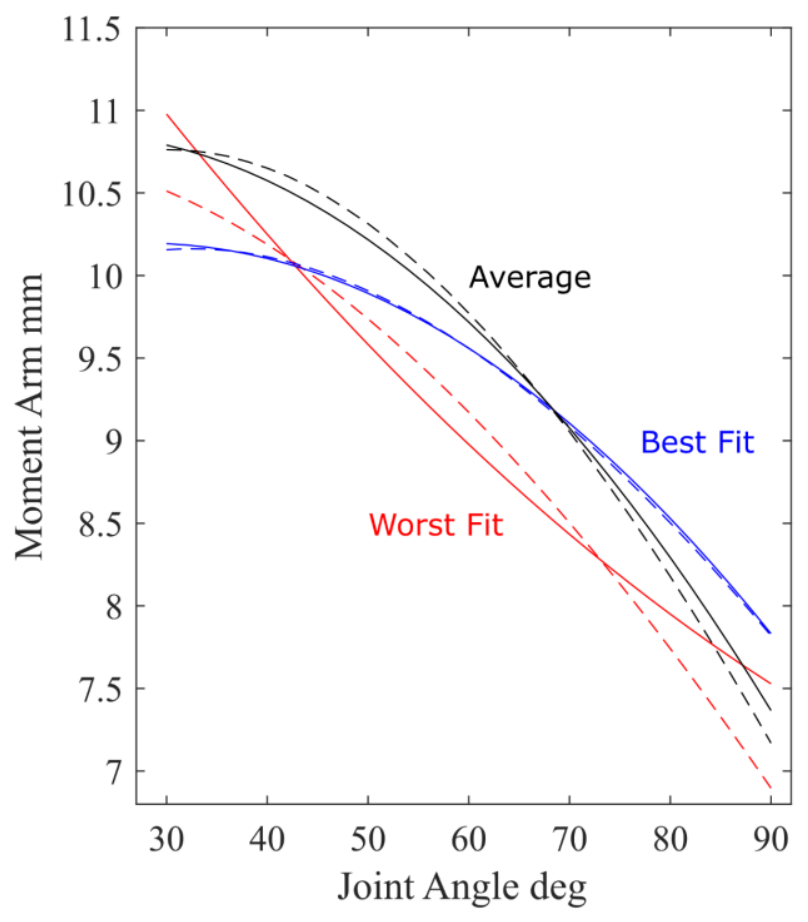

B

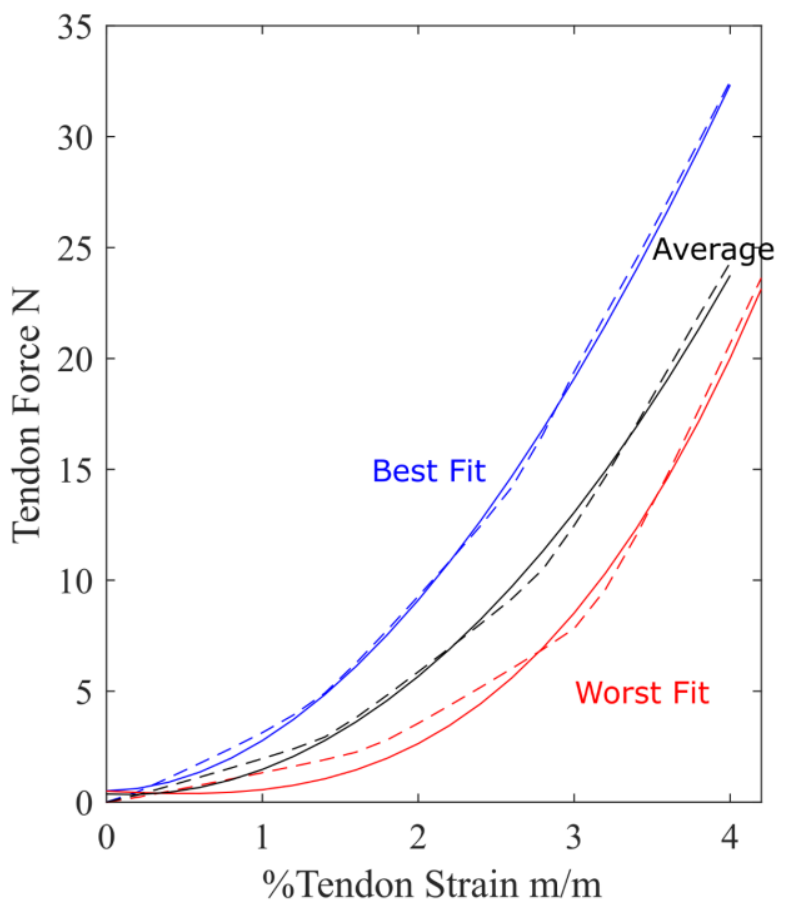

Figure S1: Example comparisons between experimental (solid lines) and modeled (dashed lines) moment arms (A) and tendon force-strain curves (B). In each plot, experimental and modeled curves are displayed for three animals, showing the best, the average and the worst fit across individuals. 
875 Additionally, the tendon force-strain curve was updated to match experimentally collected force-

876 strain values. Because OpenSim scales the tendon force-strain curve by the maximum isometric

877 force of the muscle, each tendon force-strain curve was normalized by the maximum isometric force

878 capacity of the LG or MG, respectively. The parameters of the Millard muscle model's tendon force-

879 strain curve were iteratively varied for both the LG and MG and compared to the experimental

880 curve for each individual, resulting in an average root mean square error (normalized by tendon

881 force) over 26-31 points for each tendon force-length curve (Figure S1B) of 0.061+0.025. 